\title{
Linkage function of monitoring and alarm system for Urban utility tunnel engineering
}

\author{
Yan Wei ${ }^{1}$, Jianxun $\mathrm{Ma}^{2}$, Bo dang ${ }^{1}$, Dong $\mathrm{Luo}^{2^{*}}$, Ningjun dang ${ }^{1}$, Junnan $\mathrm{Li}^{2}$ and \\ Yuanyuan $\mathrm{Li}^{2}$ \\ ${ }^{1}$ Power China Northwest Engineering Corporation Limited, Xi'an 710065, China \\ ${ }^{2}$ Department of civil engineering, Xi'an Jiaotong University, Xi'an 710054, China \\ luodong@xjtu.edu.cn
}

\begin{abstract}
Keywords: Urban utility tunnel engineering; Monitoring and alarm system; Linkage function.
Abstract. The perfect monitoring and alarming system in the urban utility tunnel is an important guarantee for the normal operation of the integrated pipe gallery. This paper first introduces the general situation of the monitoring and alarm systems in the monitoring and alarm system of the urban utility tunnel, then systematically expounds the interaction between the subsystems, and finally analyzes the linkage function of the system to provide the basis for the accuracy and reliability of the monitoring system. Thus preventing accidents, ensuring urban operation and improving economic benefits.
\end{abstract}

\section{Introduction}

Urban municipal tunnel engineering is a public facility built in the city underground for laying municipal public pipelines. It sets up the power cable, information electricity cable, thermal pipeline, pipe water, gas pipeline and other municipal engineering pipelines, and the corresponding overhaul and feeding port will be set up at a certain distance. A complete and complete monitoring system and unified planning, design, construction and management are important infrastructures to ensure the daily operation of the city [1].

The monitoring and warning system of integrated pipe gallery is divided into environment and equipment monitoring system, safety precaution system, communication system, alarm system, etc. In order to ensure normal operation of pipeline and control equipment in pipe gallery, it can react quickly in case of accidents. As the nerve center of the safety management and control system of the whole utility tunnel, the utility tunnel monitoring center passes the automatic monitoring and detection equipment to collect the information of any corner in the tube Gallery quickly and collect it in the monitoring center so that the managers can easily grasp all the conditions at any time. Establish a perfect warning and alarm mechanism to solve the underground hidden danger of urban utility tunnel facilities were destroyed by human, and ensure the normal operation of ventilation, lighting, drainage, fire prevention and communication equipment in the utility tunnel [2].

Integrated utility tunnel monitoring and alarm system is a very comprehensive management and control system. Because of the different technical system and the different standards, it is difficult to be compatible with each other. It not only needs to take account of the requirements of environment and equipment monitoring, communication and geographic information, but also needs to take account of the needs of disaster accident early warning, security prevention and so on. At the same time, the alarm and door need to be considered. The integration of the supporting system and the linkage with the broadcasting system. Therefore, in the process of interconnection of these systems, there is bound to be a heterogeneous island of information. To break the situation of information island, the monitoring and alarm system of complex and heterogeneous mode needs to solve the compatibility of different manufacturers and different products and the inter integration of multiple systems. It is necessary to consider the network access of the platform and the way of resource development. It also needs to be designed based on the Perspective of large network users to grasp the mutual benefit and share. The principle. Therefore, it is of great significance to study the monitoring and warning system of integrated utility tunnel. 


\section{Monitoring and Alarm System}

\section{The environment and equipment monitoring system.}

The function of the environment and equipment monitoring system is to realize the whole process monitoring of the parameters and state of the environment and equipment in the comprehensive utility tunnel, and transmit the real-time monitoring information to the unified management information platform of the monitoring center accurately and promptly through the multi-function integrated management and control division station, so that the personnel on duty can find the scene environment and set up in time. Prepare problems, troubleshooting and timely handling of police information to ensure normal operation of utility tunnel [4].

The integrated utility tunnel environment and equipment monitoring system consists of 4 subsystems: environmental monitoring, lighting control, equipment control and expansion interface. The environmental monitoring subsystem includes methane monitoring, hydrogen sulfide monitoring, oxygen monitoring, temperature monitoring, humidity monitoring and water level monitoring unit, and the equipment control subsystem is monitored by the ventilation equipment. The control and drainage pump unit and the electrical equipment monitoring unit are made up. The system function depends on the equipment monitoring system workstation, the industrial Ethernet communication module, the field control unit (PLC), the intelligent sensor, the field equipment instrument and the field equipment feedback switch device.

\section{Safety precaution system.}

The integrated safety management system monitors and manages the subsystems in a unified way. The equipment is arranged in the control center of the utility tunnel and is designed by the section of the control center of the utility tunnel. Each subsystem is designed independently, and the failure of the security management system does not affect the operation of each subsystem. The failure of a subsystem does not affect the operation of other subsystems [5].

As a lifeline of the city, the comprehensive pipe gallery is very important. Therefore, it is necessary to set up a security system to prevent the destruction of the utility tunnel, which is also the demand for daily management and control. The security system is an integrated security system composed of video surveillance system, intrusion alarm system, entry control system and electronic patrol management system. It can be integrated under one platform. The system adopts structural, standardized, modular and integrated configuration to form an advanced, reliable, economical, applicable and supporting security protection system. The fire safety monitoring center is located in the utility tunnel control center which is a restricted area with an emergency alarm device and a communication interface with the alarm of the upper level alarm center.

\section{Alarm system.}

According to the characteristic alarm system of all kinds of pipeline accidents, some detectors are set up accordingly when the detector monitors the pipeline accident. It takes measures to quickly deal with the pipeline accidents so as to avoid the other normal running pipelines. For example, when the pipe is set to the pipe, once the tube is monitored, the unit closes the relevant valve in the non normal liquid level notification line unit, and if the fire detector is set for the cable, once the cable fire is detected, the fire extinguishing equipment is quickly started. Pipeline accident refers to the accident that causes damage to the public environment of the utility tunnel and does not include the faults in the pipeline system, such as power outage, water pipe stop, communication interruption, etc.

\section{Communication system.}

The construction of the communication system is to create a good information application environment for the use and management, to receive, exchange, transmit, store, retrieve and realities of all kinds of information, and to provide facilities for the combination of all kinds of information equipment systems that are in line with the functions of information technology.

There is a need for communication between inspectors and control centers in the operation management of urban utility tunnel. Communication system is particularly important for operation and maintenance of integrated utility tunnel. Considering that the utility tunnel is underground with long distance, communication is difficult to achieve inside and outside of the tunnel. At present, it is 
adopted to choose the fixed cable communication and the space public wireless communication in parallel according to the actual demand.

\section{Linkage Control}

The detection and alarm system of the integrated pipe gallery is not enough, and it is necessary to cooperate with the corresponding linkage function to achieve effective results. The linkage between the monitoring system of the environment and equipment and the ventilation equipment, the linkage of the environment and equipment monitoring system and the drainage pump, the direct linkage of the safety guard system, the linkage between the security guard system and the other systems, the linkage between the video surveillance and the fire automatic alarm system, the automatic fire alarm and the fire fighting linkage System. The site management and maintenance of the urban comprehensive utility tunnel is relatively difficult, and the establishment of a reliable linkage control mechanism can effectively reduce the probability of reducing the fault in the field manual maintenance work.

\section{Linkage between environment and equipment monitoring system and ventilation equipment.}

Through the integrated monitoring and control system software platform, the monitoring and positioning of the environment and equipment monitoring system, the integration of multi system with the ventilation equipment, the emergency broadcasting system and other systems are realized. It includes the setting of the emergency linkage function of the toxic and harmful gas such as methane and hydrogen sulfide, the selection of sensor node number, the selection of the location division and the selection of the broadcasting station, and the integrated monitoring platform automatically starts the ventilation equipment in this section. The monitoring system software pushes the emergency linkage conditions. On the basis of the specific environmental monitoring in the utility tunnel, the monitoring system pushes the linkage information on the basis of the specific environmental monitoring in the utility tunnel. When the oxygen content, temperature, humidity, and toxic and harmful gas concentration in a certain section of the fire area exceed or lower than the set threshold, the detection instrument sends out the alarm signal. At the same time, the ventilation equipment of the section is automatically started, and the ventilation is forced to ensure the safety of the staff and the facilities in the utility tunnel. When the security system receives the linkage signal of the combustible gas alarm system, it should switch the scene of the alarm scene to the specified image display device.

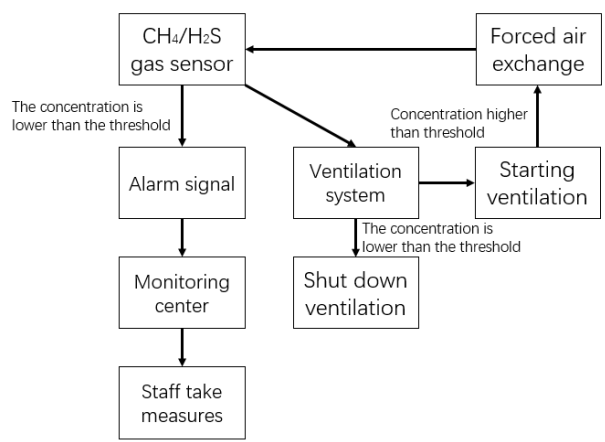

Fig. 1 linkage implementation flow diagram

\section{Linkage of environment and equipment monitoring system and drainage pump.}

In the pipe gallery, the water level switch in the water pit, the pump control box on the spot automatically starts the water pump according to the alarm signal of the liquid level. The water level of the water accumulation pit is kept under the alert level. When the liquid level in the sump exceeds the super high liquid level, the alerts are sent to the monitoring center, so that the staff can take other measures to deal with the accident. An explosion-proof liquid level gauge is set in the gas tank, the pump is started or stopped according to the height of the liquid level [6]. 


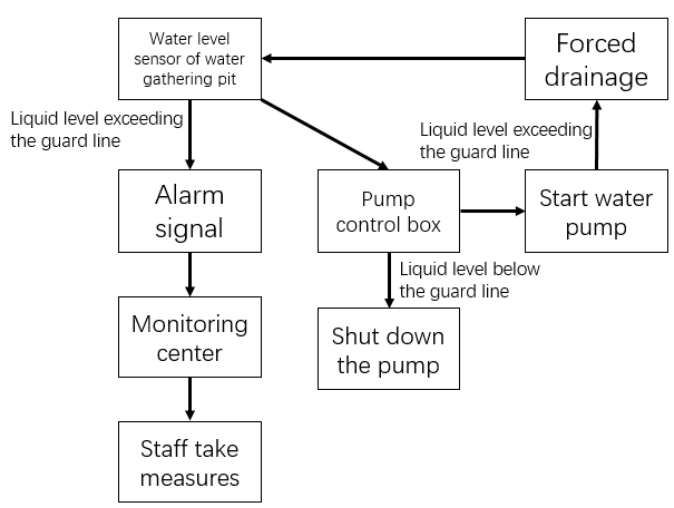

Fig. 2 flow chart of linkage system between environment and equipment monitoring system and drainage pump

\section{Linkage design between the internal security system.}

(1) Linkage between intrusion alarm system and video surveillance system.

When the illegal personnel enter the utility tunnel through the hoisting port through the external public area, the alarm management software outputs linkage signals to the corresponding video camera while enters the utility tunnel. The video camera is rotated to the predetermined position to be videotaped, video signal of the camera is cut to the main monitor and the related lighting electricity is used. The source, acousto-optic alarm and broadcasting system are released.

When multiple alarm signals appear, the monitoring of the alarm signal can be switched to different monitors in sequence. After the alarm removed, the image is cancelled automatically to prevent the false alarm.

(2) Linkage between the control system and the video surveillance system.

When someone enters the equipment room and other important areas to read the card, the entrance and exit control system can link the video surveillance system to store the scene image of the camera.

On special occasions, when the door needs to be approved by the security personnel, the video surveillance system of the exit and entry control system is used to display the camera scene image to the large screen display of the command and dispatch monitoring center, and the security personnel can open the entrance guard system through the card reader.

(3) Linkage between human intrusion warning system and entrance control system.

If the illegal cardholder is detected, the video surveillance system of the entrance control entrance control system is used to switch the scene image of the camera to the large screen display on the command and dispatch monitoring center. When the intrusion alarm system is confirmed, the intrusion alarm system can link out the entry control system, close the specified entry and exit according to the program, and can only be opened by the security personnel.

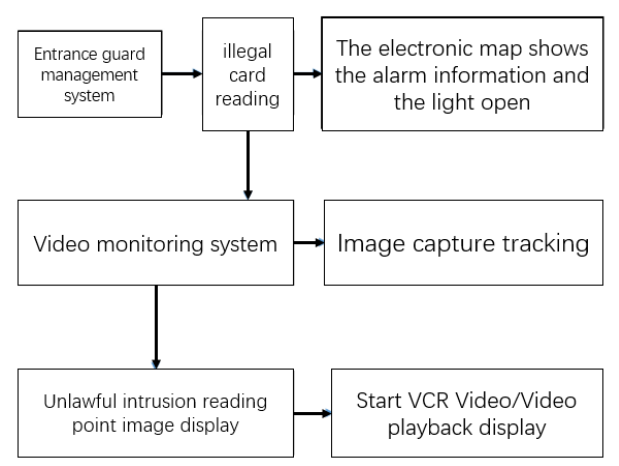

Fig. 3 Schematic diagram of linkage between access control system and video surveillance system 


\section{Linkage between the security guard system and other systems.}

According to the security guard linkage and alarm requirements, the whole system is divided into four subsystems, such as anti-theft alarm system, video surveillance system, door control system, temperature and humidity monitoring system and other auxiliary systems. All of the subsystems are connected to the monitoring center through the network.

When the security system alarm or receive the linkage signal of the environment and equipment monitoring system and the automatic fire alarm system, the lighting of the alarm scene should be opened and the scene picture of the alarm is switched to the designated image display device.

(1) Linkage between intrusion alarm system and intelligent lighting control.

When illegal personnel enter the inside of the gallery through the entrance and outlet of the outdoor public area, the intrusion alarm system can link the intelligent lighting control system and open the lighting of the field area.

(2) Linkage between access control system and lighting equipment.

When someone enters the inspection hall, the entrance control system can be linked with the lighting control system to turn on the lights in the scene area.

(3) The linkage between exit and entry control systems and systems.

The control device of the entrance and exit should be linked with the monitoring system of the environment and equipment and the automatic fire alarm system. In the case of emergency, the locking state of the corresponding exit and exit control device should be linked together.

\section{Fire automatic alarm and fire fighting linkage system.}

The main part of the integrated pipe gallery is the main pipeline, which is responsible for the normal operation of the city or area. Once the fire is not discovered and extinguished in time, the infrastructure of the city or the area will be paralyzed, and the city is subjected to huge economic loss and bad social influence. Therefore, it is very necessary to set up fire monitoring, automatic alarm and linkage fire extinguishing system [7].

(1) Fire automatic alarm system

The automatic fire alarm system consists of three parts: detector, signal circuit and automatic alarm device. There are many kinds of fire detectors, such as ion smoke detectors. Photoelectric smoke detector. temperature detector (including fixed temperature and differential temperature).

At present, the automatic alarm devices used in production and use are mostly used in multi line system, which are divided into regional alarm controller, centralized controller and intelligent fire alarm controller. The regional alarm system is composed of a regional fire alarm controller and a fire detector. It is composed of a fire alarm controller and a fire detector. The system is characterized by simple function and wide use. It is suitable for projects with small engineering scale, few fire detection areas and small protection range, mostly local protection, and is suitable for comprehensive pipe gallery projects.

Fire alarm system is a fire alarm system composed of trigger device, fire alarm device, fire alarm device and other auxiliary equipment. In order to detect the fire and take effective measures in time to control and extinguish the fire, the fire alarm system is set in the tube corridor.

(2) Control module of fire fighting linkage control system

There are various types of input and output control modules and signal modules in fire alarm and linkage fire extinguishing systems. The function of the control module is to control the opening and closing of all kinds of linkage equipment. The function of the signal module is to feed the action signals of various switches to the alarm, and to display the position of the switch on the alarm, so as to understand the location of the fire field. The function of the control module is as follows:

1) The control and display function of hydrant system: control the opening and closing of fire pumps. The position of the pump button is displayed to show the work and fault state of the fire pump.

2) Automatic sprinkler system's control and display function: control the automatic fire sprinkler system's opening and closing. It shows the working state of the spray pump, and the fault state shows the working state of the alarm valve, gate valve and water flow indicator. 
3) The control display function of the carbon dioxide gas automatic fire extinguishing system: control the emergency start and cut function of the carbon dioxide gas fire extinguishing system, control the linkage of the fire detector with the 30s adjustable delay function, showing the function of the manual and automatic working state of the carbon dioxide gas fire extinguishing system in the alarm, At each stage of the injection, the control room should have the corresponding acousto-optic alarm signal, and the sound signal can be removed manually. During the delay phase, fire doors and windows should be closed, and ventilation and air conditioning systems should be stopped.

4) The function of the fire control control equipment to the linkage control object: after the fire alarm, stop the fan to close the fire valve, and receive the feedback signal function fire confirmation, close the fire door and the fire curtain of the relevant parts.

5) After the fire is confirmed, the function of connecting the fire emergency lamp and the evacuation indicating lamp has the function of cutting off the non fire fighting power.

(2) Fire monitoring automatic control and fire fighting linkage process

1) The alarm signal of two independent fire detectors in the same protection area, a fire detector and a manual fire alarm button, or the emergency start signal outside the protection area, should be used as the linkage trigger signal of the system.

2)After receiving the first linkage trigger signal that meets the linkage logic relationship, the fire controller starts the fire acousto-optic alarm set in the protection area, and sends out the linkage control signal after receiving the second linkage triggering signals, and the linkage trigger signal is the fire detection in the protection area and the first alarm. The alarm signal of a temperature sensitive fire detector or a manual alarm button adjacent to a manual or manual fire alarm button.

3) Linkage control signals should include the following: close the fire protection valve and ventilator in the protection area, and start the ultra-fine dry powder extinguishing device.

4) The fire acousto-optic alarms of the gas sprinkler are set up above the exit of the gas fire protection zone. The sound signal indicating the release of the gas should be distinctly different from the sound signal of the fire sound alarm set in the protection object. When starting the gas fire extinguishing device, start the fire sound and light alarm set at the entrance of the protective area to indicate the gas spray.

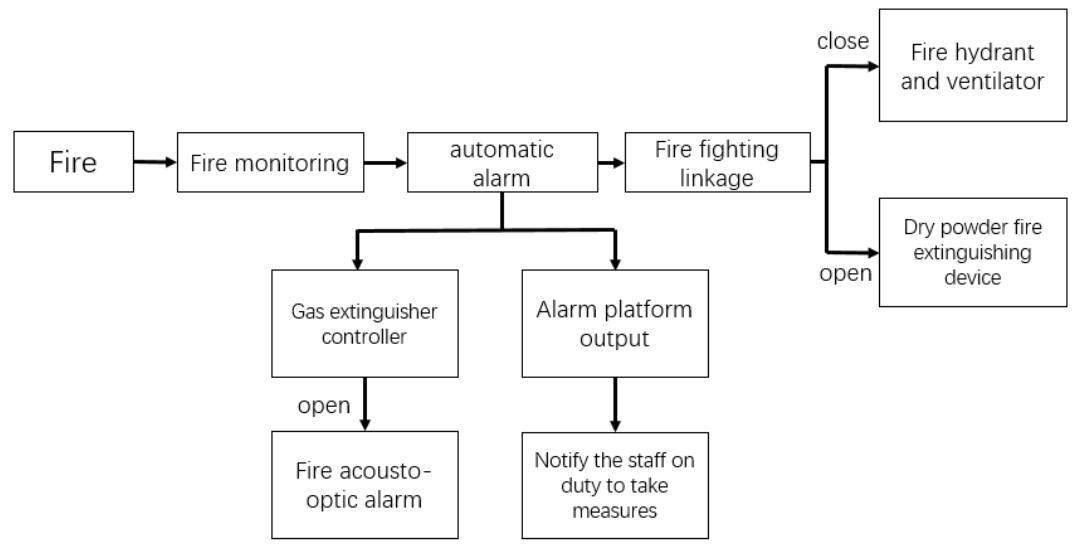

Fig. 4 flow chart of automatic fire alarm and fire fighting linkage system

5) Because the system's gas fire control controller does not directly connect the fire detector, the linkage trigger signal of the gas fire extinguishing system is issued by the fire alarm controller or the fire control linkage controller.

6) The linkage control and feedback signals of each stage of the superfine dry powder extinguishing device are fed back to the fire linkage controller.

7) After the fire is extinguished, the post-accident ventilation started. Staff will be allowed to enter after the the air targets monitored by the environmental monitoring system meet the requirements. 


\section{Conclusions}

The perfect monitoring and alarm system is an important guarantee for the normal operation of a comprehensive corridor, so the corresponding monitoring system must also have accurate line and reliability to prevent accidents, guarantee the operation of the city and improve the economic benefit. The problem of how to solve the early warning and processing, improve the communication system and the integration and integration of various systems based on the monitoring and alarm system of the Internet of things is the future development direction of the integrated corridor monitoring and alarm system.

\section{Acknowledgements}

This work was supported by Research on key technology of urban utility tunnel design under China Electric Power Construction Group northwest investigation and Design Research Institute Co., Ltd.(20180337), National Natural Science Foundation of China (No. 51508460), Central University's special research funding special fund cross disciplinary project (xjj2017175), Natural Science Foundation Research Program of Shaanxi Province - Youth Talents Project (2016JQ5017), National Post-Doctoral Science Foundation (No. 2015M570838), Shaanxi Province Post-Doctoral Research Fund (2016BSHB083) , Shaanxi Province Post-Doctoral supporting research project (2015SXBHPT112), Research Fund Project of Xi'an Jiao Tong University 。

\section{References}

[1] R. Li, H.C. Fu. Design of integrated pipe gallery monitoring and alarm system. [J]. Intelligent Building Electrical Technology, 2016, 10 (3): 67-70.

[2] G.F. Kang. Analysis of the development status and trend of underground integrated pipe gallery in the construction of smart city [J]. Railway Building Technology, 2016 (z1).

[3] Y. Zhuang, W. Zhang. Monitoring and emergency broadcasting system to achieve linkage [J]. Coal Mine Safety, 2018, 49 (1): 126-128.

[4] H. Zhang. Wisdom monitoring and warning system for integrated pipe corridors [J]. Construction Technology, 2016 (21): 20-22.

[5] K. Yang, Da. W. Zhang. Design of monitoring and alarm system for underground utility tunnel [J]. Intelligent Building, 2017 (6): 31-33.

[6] X. Zhang, C. Yang, X. Zhang. Design of monitoring and alarm system for 110 National Road Corridor in Baotou, [J]. Digital Technology and Application, 2017 (10): 149-152.

[7] G. Zhao, X. Wang, Z. Hu. Design of automatic fire alarm and fire fighting linkage system [J]. Intelligent Building and Urban Information, 2006 (1): 94-97. 